\title{
Intestino e rene. La riscoperta di un antico legame
}

\author{
Angela Maria Pellegrino ${ }^{1}$, Luigi Annicchiarico Petruzzelli ${ }^{1}$, Antonietta Ascione ${ }^{1}$, Antonella Caiazza ${ }^{1}$, Francesco Cappellaio $^{1}$, \\ Andrea Memoli ${ }^{1}$, Laura Pirro ${ }^{1}$, Luigi Russo², Bernadette Scognamiglioํㅜ, Domenico Russo ${ }^{1}$ \\ ${ }^{1}$ Dipartimento di Sanità Pubblica, Unità di Nefrologia ed Ipertensione, Università degli Studi di Napoli Federico II, Napoli \\ ${ }^{2}$ Seconda Università di Napoli, Unità di Nefrologia, Napoli
}

\section{Crosstalk between intestine and kidney. Revival of an ancient link}

Chronic kidney disease (CKD) is associated with systemic inflammation. The latter accelerates comorbidities that are associated with CKD (cardiovascular disease, anemia, infections, cachexia and several other conditions), shortens life span and greatly impairs the quality of life.

Recently the attention has been focused intestine and intestinal microbiota as sources of systemic inflammation. On one hand, the microbiota promotes the production of uraemic solute with a biological impact on the progression of kidney failure and vascular diseases. On the other hand, the uraemic status alters the composition of the intestinal microbiota and the structure of the epithelial barrier, causing the translocation of both microbiota and uraemic toxins into the blood stream.

Several approaches have recently been tested, including dietary measures, prebiotics, synbiotics and intestinal sorbents in order to reduce the generation and serum concentration of uraemic toxins.

Keywords: Inflammation, Intestine, Bacterial microflora, Prebiotics, Intestinal sorbents

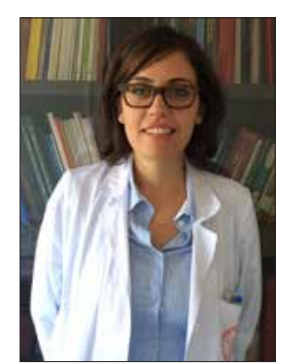

Angela Maria Pellegrino

\section{Introduzione}

L'infiammazione gioca un ruolo chiave nella progressione della malattia renale cronica (MRC) e delle sue maggiori complicanze, come la malattia cardiovascolare e la malnutrizione, che sono due forti predittori di mortalità e morbidità (1). Un'attivazione infiammatoria è stata riportata nel $30-50 \%$ dei pazienti con $\operatorname{MRC}(2,3)$.

I maggiori fattori che contribuiscono allo stato infiammatorio sono le tossine uremiche, le infezioni, le malattie autoimmuni, l'ipertensione e l'ipervolemia (4). Recentemente è stato riconsiderato l'importante ruolo dell'intestino e della sua flora batterica come fonti di infiammazione in corso di MRC (5).

La maggior parte degli epiteli di superficie del nostro corpo, come la cute e le mucose, è colonizzata da microrganismi che

Accepted: May 23, 2015

Published online: June 15, 2015

Indirizzo per la corrispondenza:

Domenico Russo

Via Pansini 5

80100 Napoli

domenicorusso51@hotmail.com rappresentano la normale microflora, il cosiddetto microbiota. II microbiota contiene trilioni di cellule batteriche, un numero tre volte superiore rispetto alle cellule che costituiscono il nostro corpo (6); dai 10 ai 100 trilioni di batteri popolano l'intestino dell'adulto (7). La mucosa intestinale, estendendosi per un'area di 300-400 m², costituisce la più ampia superficie di contatto tra l'ospite e l'ambiente esterno ricco di stimoli antigenici, tossici e mitogeni presenti nel cibo e nel microbiota stesso (7).

La funzione di barriera della mucosa intestinale è garantita da complessi meccanismi che agiscono su vari livelli: la barriera ecologica (la normale flora batterica), la barriera meccanica (singolo strato di cellule intestinali polarizzate, gli enterociti, coperte da muco) e la barriera immune (IgA secretorie, cellule del sistema immune come i macrofagi, i neutrofili, i natural killer, le placche di Peyer e i linfonodi mesenterici) (7). Circa l' $80 \%$ delle cellule immunologicamente attive che compongono il nostro sistema immune è presente nel tratto gastrointestinale (6). In condizioni fisiologiche, tutti questi sistemi garantiscono la simbiosi tra l'ospite e il microbiota, limitando un'eccessiva proliferazione batterica (8).

I microrganismi che popolano la superficie intestinale esplicano funzioni essenziali: metabolismo di xenobiotici (dal greco xénos, estraneo: le sostanze estranee all'organismo che possono causare effetti biologici indesiderati), protezione contro i patogeni, rinnovamento dell'epitelio intestinale, generazione di vitamine come la vitamina $\mathrm{K}$, la cobalamina, la tiamina e la riboflavina e bilanciamento della funzione immunitaria (5). 
Il passaggio di tossine dal lume intestinale al sangue avviene attraverso due vie: la via para-cellulare, che, attraverso le tight-junction, controlla il flusso tra due cellule epiteliali contigue, e la via trans-cellulare, che è regolata da pompe e canali specifici di membrana. L'integrità di tale sistema evita la traslocazione delle tossine batteriche dall'intestino al circolo ematico.

L'endotossina batterica è un lipopolisaccaride (LPS), il maggiore componente della parete esterna dei batteri Gramnegativi, che rappresentano circa il $70 \%$ dei batteri presenti nell'intestino. L'esposizione all'endotossina rappresenta un importante stimolo pro-infiammatorio, che determina il rilascio di citochine e, attraverso il CD14, anche l'attivazione di cellule immuno-competenti (9).

Numerose malattie possono determinare un'alterazione della barriera fisica o dell'immunità intestinale, favorendo la traslocazione batterica (10):

- le malattie infiammatorie croniche intestinali

- la proliferazione batterica indotta da farmaci o da infezioni

- la terapia radiante sull'addome

- la malnutrizione con conseguente atrofia della mucosa

- la nutrizione parenterale

- la sepsi da Gram-negativi (danno della parete intestinale provocato dal rilascio di ossido nitrico)

- lo scompenso cardiaco per l'edema della parete intestinale

- la MRC

L'intestino e la MRC si influenzano a vicenda in una relazione bidirezionale. Da un lato, il microbiota intestinale influisce sulla sintesi di tossine uremiche con un forte impatto negativo sul danno vascolare e sulla progressione della MRC. Dall'altro, lo stato uremico influisce sulla composizione del microbiota intestinale e determina un danno tossico-ischemico della barriera intestinale con conseguente traslocazione di endotossine $(5,11)$.

Infatti, le alterazioni metaboliche presenti nei pazienti con MRC incidono negativamente sull'equilibrio tra il microbiota e i patogeni, favorendo la crescita di questi ultimi, condizione nota con il nome di disbiosi. L'accumulo dell'urea nei fluidi extracellulari e la sua diffusione nel tratto gastrointestinale determinano l'espansione delle specie microbiche produttrici di ureasi, che formano ammonio e idrossido di ammonio, prodotti in grado di danneggiare le tight-junction e di attivare la cascate dell'infiammazione. Nel corso della MRC è stato osservato che i livelli di endotossinemia aumentano progressivamente con il ridursi della funzione renale, raggiungendo la loro maggiore concentrazione allo stadio $\mathrm{V}$; i livelli di endotossine sieriche aumentano ulteriormente nei pazienti in trattamento sostitutivo (senza sostanziali differenze tra la dialisi extracorporea e quella peritoneale) (Fig. 1) (9).

Per quanto riguarda l'associazione tra endotossinemia e infiammazione, è stata osservata una correlazione tra livelli

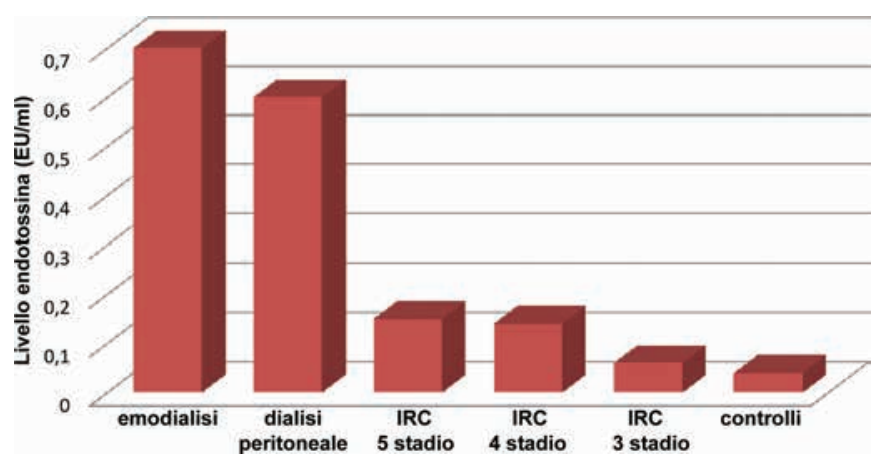

Fig. 1 - Livelli di endotossina circolante nei vari stadi della malattia renale cronica e nei controlli. L'endotossinemia aumenta progressivamente con il peggioramento della funzione renale raggiungendo concentrazioni più elevate nei pazienti in trattamento dialitico sostitutivo, senza sostanziali differenze tra la dialisi extracorporea e la dialisi peritoneale.

sierici di LPS e concentrazione plasmatica di proteina $C$ reattiva (PCR), spessore medio intimale carotideo (IMT, valutato mediante Doppler) e valori di albuminemia. L'endotossinemia (misurata come concentrazione di LPS in 30 pazienti incidenti in dialisi peritoneale) risultava significativamente più alta rispetto a quella dei pazienti con MRC, a quella dei pazienti con nefropatia da IGA e a quella dei controlli. Inoltre, la concentrazione di LPS era direttamente correlata con i valori di PCR e con I'IMT carotideo e inversamente correlata con i valori di albuminemia. Per quanto singola, questa evidenza può avvalorare l'ipotesi che l'endotossinemia possa attivare l'infiammazione sistemica ed essere correlata con un'accelerazione dell'aterosclerosi (4).

Nella MRC, oltre alla disbiosi, è presente un importante danno della barriera intestinale, causato dall'ipervolemia che caratterizza quasi tutte le fasi della MRC. La ritenzione di fluidi causa un edema generalizzato della parete intestinale, con alterazione della funzione epiteliale di barriera. Nei pazienti in emodialisi cronica, inoltre, si aggiunge un terzo fattore: I'instabilità vascolare con la rapida sottrazione di fluidi. L'ipotensione intra e post-dialitica causa ischemia della parete intestinale con ulteriore traslocazione di endotossine batteriche. Questo processo attiva l'immunità innata con conseguente produzione di citochine pro-infiammatorie, le quali amplificano a loro volta la disfunzione della barriera (10). Infatti, aumentati livelli di endotossinemia sono stati associati al sovraccarico di fluidi, dovuto all'incapacità del rene a eliminare acqua e soluti. In presenza di espansione del volume extracellulare (valutato mediante misurazione ecografica del diametro della vena cava inferiore e del relativo indice di collassabilità), si osservava un aumentato livello sierico di endotossine (12) in pazienti con MRC non in dialisi.

Il ruolo della volemia sul livello di endotossine è stato confermato anche nei pazienti in trattamento extracorporeo (9). La concentrazione di endotossine nel post-dialisi si correlava 
con la quantità di fluidi rimossi durante la seduta e con la riduzione della pressione arteriosa sistolica registrata durante l'emodialisi. Inoltre, l'endotossimenia era strettamente correlata con i valori di PCR e con una ridotta sopravvivenza a un anno (Fig. 2) (9).

Questi dati confermano l'associazione tra endotossinemia, volemia e infiammazione; essi indicano, inoltre, che, nei pazienti in dialisi extracorporea, la traslocazione batterica sia maggiore per l'effetto combinato dell'ipervolemia e della rapida rimozione del volume, responsabile di instabilità vascolare e di ischemia della mucosa intestinale per una riduzione della perfusione splancnica (9).

\section{Potenziali interventi}

Molte tossine uremiche sono prodotte nell'intestino dalla fermentazione batterica. Aminoacidi come il triptofano e la tirosina vengono trasformati dal microbiota nei precursori delle tossine uremiche: indolo, p-cresolo, fenoli, ippurati, trimetilamine. Nella MRC numerose condizioni favoriscono la formazione di queste tossine. Gli amminoacidi, se poco assorbiti lungo il tratto gastrointestinale, rappresentano un ricco substrato per il metabolismo microbico. Nella MRC, la motilità intestinale è fortemente rallentata, inducendo la proliferazione dei batteri proteolitici e un aumento del tempo di contatto di tali tossine con la mucosa (5). Essendo le proteine la fonte di amminoacidi, la riduzione del loro introito porta a una minore sintesi di tossine uremiche. Per quanto una dieta ipoproteica abbia un effetto nefro-protettivo, bisogna tenere presente il fatto che numerosi pazienti con MRC presentano segni di malnutrizione, complicanza che aumenta il rischio di morbidità e mortalità. Per questo è necessario che l'obiettivo della riduzione delle tossine mediante una dieta ipoproteica tenga conto anche dell'apporto calorico e della compliance dei pazienti.

Un ulteriore approccio terapeutico può essere rappresentato dalla riduzione della sintesi di tossine uremiche, modificando il microambiente intestinale. In tal senso agisce la somministrazione di prebíotici, probiotici e simbiotici. I probiotici sono microrganismi vivi presenti nei cibi o somministrati come tali, che migliorano la composizione e la funzione della flora intestinale. I prebiotici sono delle sostanze non digeribili presenti nei cibi che stimolano la crescita di specifiche specie batteriche favorevoli per l'uomo. I simbiotici contengono un misto di probiotici e prebiotici. II trattamento con una formulazione di simbiotici in pazienti con fasi non avanzate di MRC ha portato a una riduzione significativa delle concentrazioni di p-cresolo (Fig. 3) (13).

Un'altra opzione terapeutica può essere rappresentata dall'impiego di "chelanti intestinali" che potrebbero rimuovere le endotossine o i loro precursori. In vitro, il sevelamer (resina sintetica comunemente impiegata come chelante del fosforo non contenente né calcio né alluminio) riduceva la concentrazione di endotossine (14). Tale effetto del sevelamer

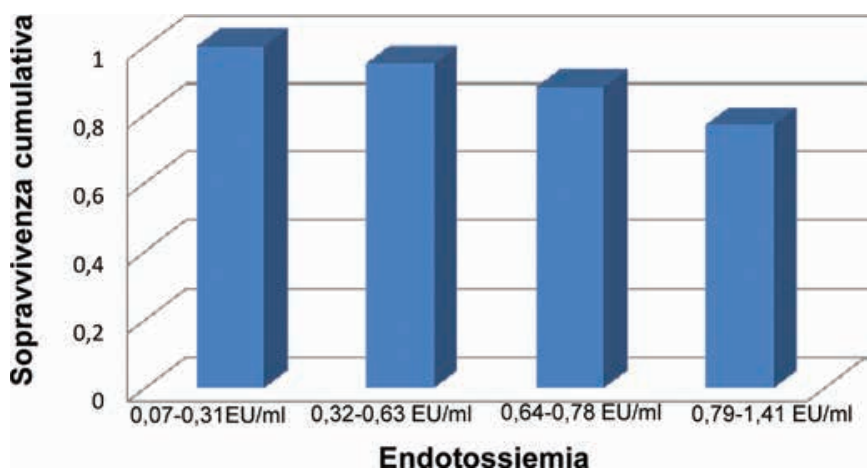

Fig. 2 -Correlazione tra valori di enodotossina circolante e sopravvivenza ad un anno in pazienti in trattamento extracorporeo. L'endotossiemia correla con una ridotta sopravvivenza ad una anno.

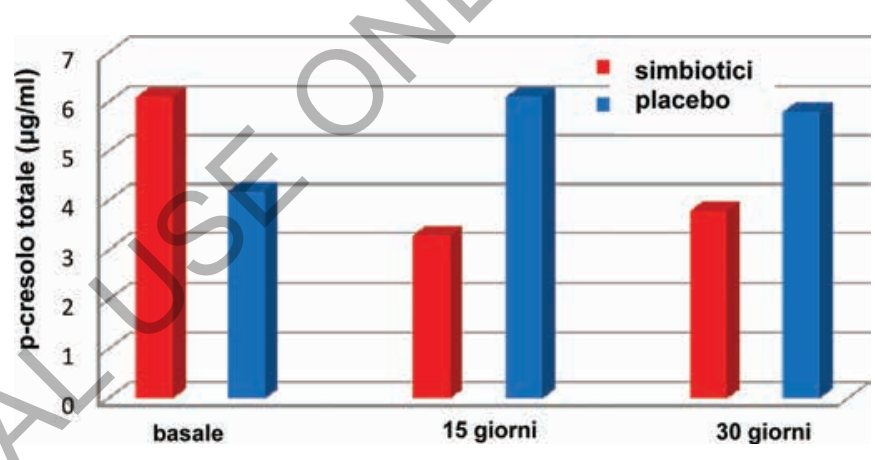

Fig. 3 - II trattamento con simbiotici in pazienti in fase non avanzata della malattia renale cronica ha portato a riduzioni significative della concentrazione plasmatica del p-cresolo (a 15 e a 30 giorni dall'inizio del trattamento).

è stato confermato in studi clinici. È stato valutato l'effetto del calcio acetato e del sevelamer sui livelli di endotossine e sugli indicatori sierici dell'infiammazione in pazienti in emodialisi. L'endotossinemia era marcatamente ridotta nel gruppo trattato con sevelamer. La concentrazione sierica di IL-6 e di PCR risultava più alta nei pazienti trattati con il calcio acetato rispetto al sevelamer (15). In un altro studio è stata misurata la concentrazione di $\mathrm{p}$-cresolo in pazienti in stadi non avanzati della MRC. La concentrazione plasmatica di p-cresolo risultava significativamente più bassa nei pazienti in trattamento con sevelamer rispetto a quella sia dei pazienti in trattamento con lantanio che dei pazienti controllo (16).

\section{Conclusioni}

- In corso di MRC si verifica un'alterazione del microbiota indotta dalle tossine uremiche.

- Nella MRC si instaura un danno tossico-ischemico della parete intestinale causato dall'espansione del volume e dall'instabilità vascolare, che contribuisce alla traslocazione batterica.

- La traslocazione batterica amplifica lo stato infiammato- 
rio in tutte le fasi della MRC.

- Esiste un'associazione tra livelli di endotossinemia e sopravvivenza.

- La riduzione dell'apporto proteico rimane una strategia terapeutica tuttora essenziale (evitando la malnutrizione).

- L'uso di prebiotici, probiotici e simbiotici migliora il microbiota intestinale riducendo la produzione di endotossine.

- L'uso di chelanti a base di resine riduce l'endotossinemia per meccanismi non dipendenti dal controllo dei parametri del metabolismo minerale.

\section{Disclosures}

Financial support: No financial support was received for this submission. Conflict of interest: The authors have no conflict of interest.

\section{Bibliografia}

1. Wanner C, Zimmermann J, Schwedler S, Metzger T. Inflammation and cardiovascular risk in dialysis patients. Kidney Int Suppl. 2002;80:99-102.

2. Stenvinkel $P$, Heimburger $O$, Paultre $F$, et al. Strong association between malnutrition, inflammation, and atherosclerosis in chronic renal failure. Kidney Int. 1999;55(5):1899-911.

3. Owen WF, Lowrie EG. C-reactive protein as an outcome predictor for maintenance hemodialysis patients. Kidney Int. 1998;54(2):627-36

4. Szeto CC, Kwan BC, Chow KM, et al. Endotoxemia is related to systemic inflammation and atherosclerosis in peritoneal dialysis patients. Clin J Am Soc Nephrol. 2008;3(2):431-6.

5. Vanholder R, Glorieux G. The intestine and the kidneys: a bad marriage can be hazardous. Clin Kidney J. 2015;8(2): 168-79.

6. Tlaskalová-Hogenová $H$, Stěpánková $R$, Kozáková $H$. The role of gut microbiota (commensal bacteria) and the mucosal barrier in the pathogenesis of inflammatory and autoimmune diseases and cancer: contribution of germ-free and gnotobiotic animal models of human diseases. Cell Mol Immunol. 2011;8(2): 110-20.

7. Kotanko $\mathrm{P}$, Carter $\mathrm{M}$, Levin NW. Intestinal bacterial microflora-a potential source of chronic inflammation in patients with chronic kidney disease. Nephrol Dial Transplant. 2006; 21(8):2057-60.

8. Anders HJ, Andersen K, Stecher B. The intestinal microbiota, a leaky gut, and abnormal immunity in kidney disease. Kidney Int. 2013;83(6):1010-6.

9. Mclntyre CW, Harrison LE, Eldehni MT, et al. Circulating endotoxemia: a novel factor in systemic inflammation and cardiovascular disease in chronic kidney disease. Clin J Am Soc. Nephrol 2011;6(1):133-41.

10. Sun PP, Perianayagam MC, Jaber BL. Endotoxin-binding affinity of sevelamer: a potential novel anti-inflammatory mechanism. Kidney Int Suppl. 2009;(114):S20-5.

11. Vaziri ND. Gut microbial translocation in the pathogenesis of systemic inflammation in patients with end-stage renal disease. Dig Dis Sci. 2014;59(9):2020-2.

12. Gonçalves S, Pecoits-Filho R, Perreto $S$, et al. Associations between renal function, volume status and endotoxaemia in chronic kidney disease patients. Nephrol Dial Transplant. 2006;21(10):2788-94.

13. Guida B, Germanò R, Trio R, et al. Effect of short-term synbiotic treatment on plasma p-cresol levels in patients with chronic renal failure: a randomized clinical trial. Nutr Metab Cardiovasc Dis. 2014;24(9):1043-9.

14. DeSmet R, Thermote F, Lameire N, Vanholder R. Sevelamer hydrochloride (Renagel) adsorbs the uremic compounds indoxyl sulfate, indole and p-cresol [Abstract]. JASN. 2004;15:505A.

15. Navarro-González JF, Mora-Fernández C, Muros de Fuentes M, Donate-Correa J, Cazaña-Pérez V, García-Pérez J. Effect of phosphate binders on serum inflammatory profile, soluble CD14, and endotoxin levels in hemodialysis patients. Clin J Am Soc Nephrol. 2011;6(9):2272-9.

16. Guida B, Cataldi M, Riccio E, et al. Plasma p-Cresol lowering effect of sevelamer in peritoneal dialysis patients: evidence from a Cross-Sectional Observational Study. PLoS One. 2013;8(8):e73558.

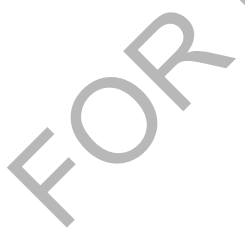

